\section{Small but long Koch fractal monopole}

\section{Puente, J. Romeu, R. Pous, J. Ramis and A. Hijazo}

A small but long wire fractal antenna based on the Koch curve is presented. Experimental and numerical results show that the antenna improves the features of a common linear monopole. The radiation resistance is increased and the $Q$ is reduced at each fractal iteration, approaching the fundamental limit on small antennas.

Introduction: It has been shown that the self-similarity property of fractal shapes can be successfully applied to the design of multiband fractal antennas $[1-4]$. It is the aim of this work to show how the space-filling properties [4] of many fractal forms can be used to design small antennas as well $[4,5]$. Experimental and numerical results are used to show how a fractal monopole can improve the radiaticn features of a common linear monopole (resonance frequency, adiation resistance, bandwidth), while some comments regarding the fundamental limit of small antennas are made.

Small antennas: Some common geometrical notions such as length, size and dimension have to be applied with great care when dealing with fractal geometry. For instance, many fractal continuous, but nowhere differentiable curves, have an infinite length, yet enclose a finite area. The classical Koch fractal curve shown in Fig. 1 is an example of such objects. Constructed after an iterative procedure, the length of the curve grows by a factor of $4 / 3$ at each stage, yet always maintains exactly the same height. The limit object obtained after an infinite number of iterations is an ideal fractal curve with no derivative at any point.

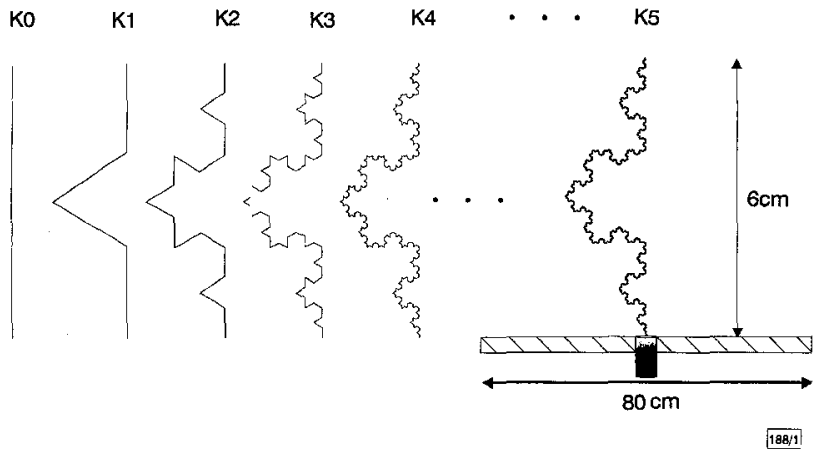

Fig. 1 Iterative construction of fractal Koch curve

The first five iterations ( $\mathrm{K} 1-\mathrm{K} 5)$ of the curve were constructed and mounted in a monopole configuration (right). A linear monopole (K0) was built under the same conditions for comparison.

Several theoretical problems arise when analysing the electromagnetic behaviour of a fractal wire antenna such as the Koch monopole in Fig. 1. For instance, the evaluation of the radiation vector through a line integral along the antenna wire is not proved to converge for the ideal fractal shape. In the case of non-convergence, one might make the striking conclusion that an arbitrarily large radiation resistance might be obtained even for a monopole much smaller (but longer) than any particular wavelength.

Several authors have dealt with the fundamental limits on small antennas [6 - 8]. Wheeler and Chu stated a lower bound for the quality factor $(Q)$ of an antenna which is considered small when it can be fitted into a radianshpere, i.e. a sphere with radius $a=\lambda$ $2 \pi$. Wheeler assumed a net inductive or capacitive behaviour on the input reactance of an small antenna to derive such a fundamental limit, while Chu used an expansion of the field into spherical modes to arrive at basically the same conclusion. However, the application of the same arguments to fractally shaped antennas is not straightforward; in particular, some fractal antennas can be resonant even when considered small, and the spherical mode series used by $\mathrm{Chu}$ is not proved for ideal fractal shapes. It could be argued that an ideal fractal antenna is not feasible, but such an ideal object can be approached with a succession of feasible shapes which increase their length (but not their size) at each iteration. Whether the behaviour of such a succession converges to the fundamental limit or not appears as an unsolved question, as suggested by the following results.

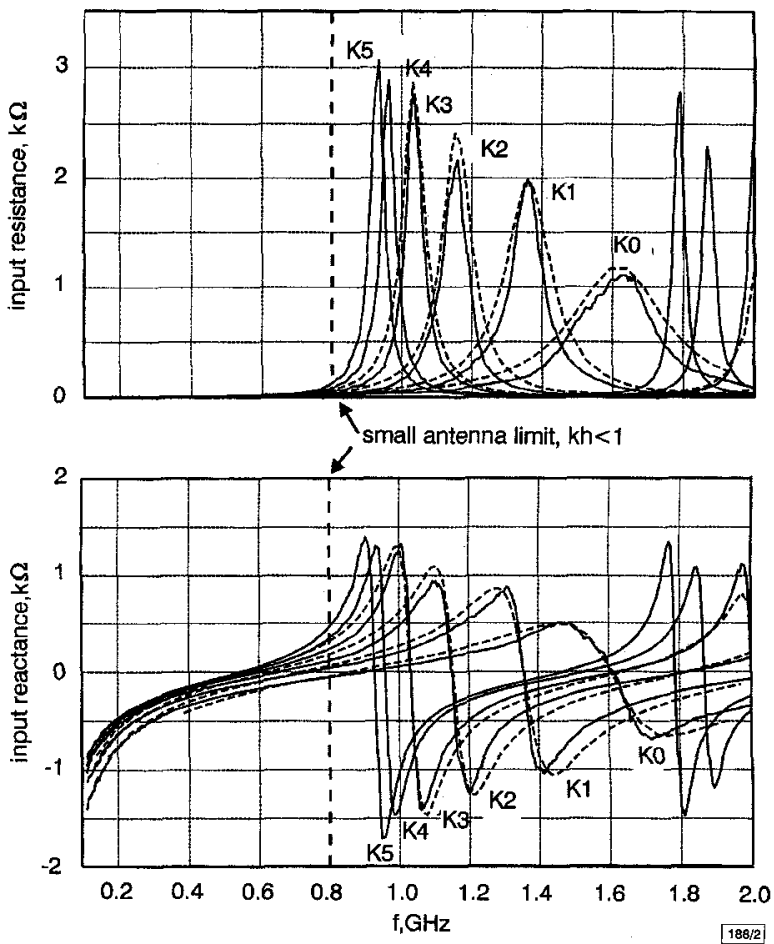

Fig. 2 Input resistance and reactance for $\mathrm{KO}-\mathrm{K} 5$ antennas

Notice the resonant behaviour of the fractal antennas below the small antenna limit. Input resistance is increased and reactance reduced at each iteration

experimental

- - computed (MoM)

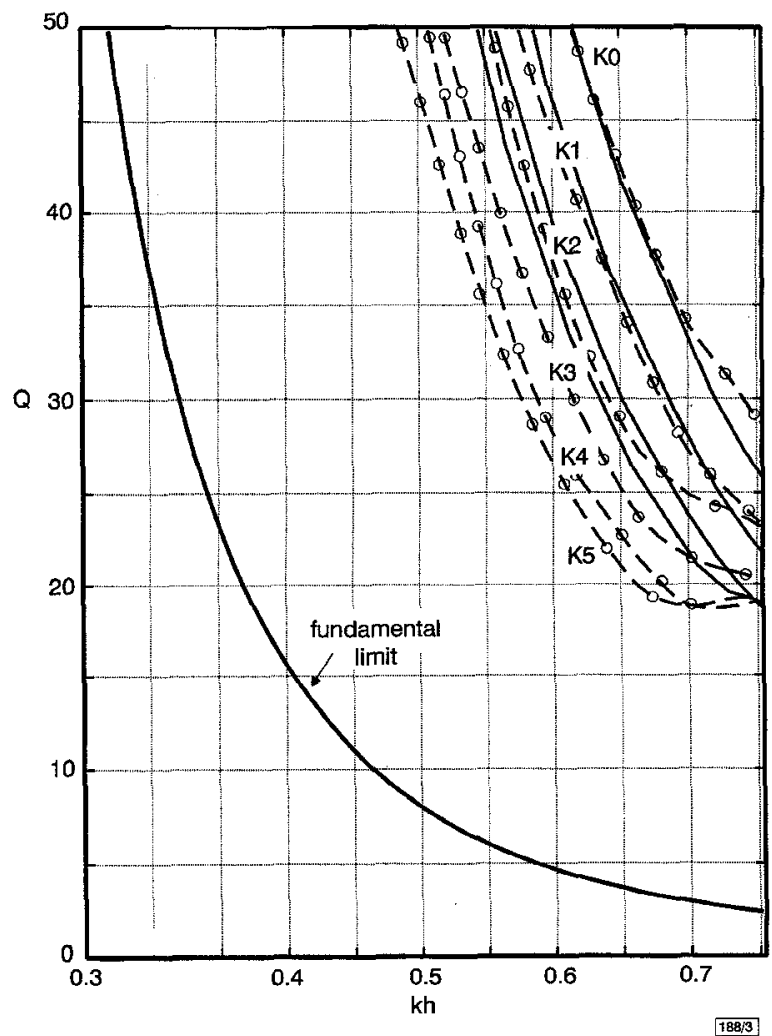

Fig. $3 Q$ factor of $\mathrm{KO}-K 5$ antennas against normalised frequency

Notice that the fractal antenna approaches the limit at each new iteration

--- experimenta

numerical

Results and discussion: Five antennas (K1 - K5) corresponding to the first five iterations of the Koch curve were implemented (Fig. 1). 
Also, a standard monopole ( $\mathrm{K} 0)$ of the same height $(h=6 \mathrm{~cm})$, but of course not the same length, was constructed for comparison. The first three iteration antennas together with the linear monopole $(\mathrm{K} 0-\mathrm{K} 3)$ were simulated using the moments method. Fig. 2 displays the input resistance and reactance of the six antennas, while their $Q$ factor with respect to the normalised frequency is shown in Fig. 3.

Some interesting conclusions can be derived from those plots. The input resistance is increased each time the length (not the size) of the antenna is increased. Although both the radiation and ohmic resistance grow, the latter is kept [4] over a wide range of frequencies below the small antenna limit frequency $(k h<1)$, at least an order of magnitude below the radiation resistance. Also, the resonance frequency is shifted towards longer wavelengths becoming resonant antennas even in the small antenna region. Analogously, the $Q$ factor (Fig. 3) is reduced at each iteration, tending to the fundamental limit. Although the ohmic losses of the antennas contribute to a reduction in the overall $Q$, the numerical results (which do not include such losses) evince the same tendency, and a comparison of experimental and numerical data show thats the ohmic losses do not play a major role in reducing the antenna $Q$.

A physical explanation of such a behaviour might be found in the increasing number (unbounded) of sharp corners and bends of the fractal monopole, which would enhance radiation. Actually, the improvement is not surprising if the space filling properties of fractal objects are taken into account; while the one dimensional linear monopole poorly uses the available volume inside the radiansphere (as stated by Hansen in [8]) the Koch monopole holding a dimension $D>1(D=1.26)$ better uses such a small space to radiate more efficiently.

Conclusions: Both experimental and numerical data show that the Koch fractal monopole can improve the behaviour of common small monopoles. The radiation resistance is increased and the reactance is reduced even in the small antenna frequency region. The $Q$ is reduced at each iteration as well, tending to the limit stated by Chu. Whether a fundamental limit or a practical, technological one exists on the performance of such antennas still appears as an unsolved question.

Acknowledgments: The authors thank P. Mayes for encouraging this work. The work is partially supported by the 'Spanish Commission of Science and Technology' under grant TIC-96-0724C06-04.

\section{(C) IEE 1998}

Electronics Letters Online No: 19980114

28 October 1997

C. Puente, J. Romen, R. Pous, J. Ramis and A. Hijazo (Electromagnetics and Photonics Engineering Group, Signal Theory and Communications Department, Universitat Politécnica de Catalunya, Gran Capità sin, Mòdul D3, 08034 Barcelona, Spain)

\section{References}

1 PUENTE, C., and POUS, R.: 'Fractal design of multiband and low sidelobe arrays', IEEE Trans., 1996, AP-44, (5), pp. 1-10

2 PUENTE, C., ROMEU, J., POUS, R., GARCIA, X., and BENÍTEZ, F.: 'Fractal multiband antenna based on the Sierpinski gasket', Electron. Lett. 1996, 32, (1), pp. 1-2

3 PUENTE, C., ROMEU, J., POUS, R., and CARdAMA, A.: 'Multiband fractal antennas and arrays', in 'Fractals in engineering' (Springer Verlag, 1997)

4 Puente, C: 'Fractal antennas'. PhD Dissertation, Dept. of Signal Theory and Communications, Universitat Politècnica de Catalunya, June 1997

5 COHEN, N., and HOHLFELD, R.G.: 'Fractal loops and the small loop approximation', Communications Quarterly, Winter 1996, pp. 77 81

6 WheELER, H.A.: 'Fundamental limitations of small antennas', Proc. IRE, 1947, pp. 1479-1488

7 CHU, L.J.: 'Physical limitations on omni-directional antennas', $J$ Appl. Phys., 1948, 19, pp. 1163-1175

8 HANSEN, R.C.: 'Fundamental limitations in antennas', Proc. IEEE, February 1981, 69, (2)

\section{Simple realisation of hysteresis chaos generator}

\section{Storace and M. Parodi}

A chaos generator based on a simple hysteretic cell is described. The cell contains two passive nonlinear resistors; the only nonlinear elements of the circuit. The most important features of the circuit are investigated. A numerical simulation is presented.

Introduction: During recent years, a large number of chaos generators have been proposed. In particular, the interest of many researchers has focused on analogue circuits, because their structural simplicity makes their realisation easy. Recently, some hysteresis-based chaos oscillators have been proposed and analysed $[1,2]$. In this Letter, an hysteresis chaos generator is presented, originating from that considered in [1]. The hysteretic part of the circuit consists of a cell which has been extensively studied by the authors for a rather general problem of hysteresis modelling $[3,4]$. The cell is made up of a linear capacitor, one linear and two nonlinear resistors.
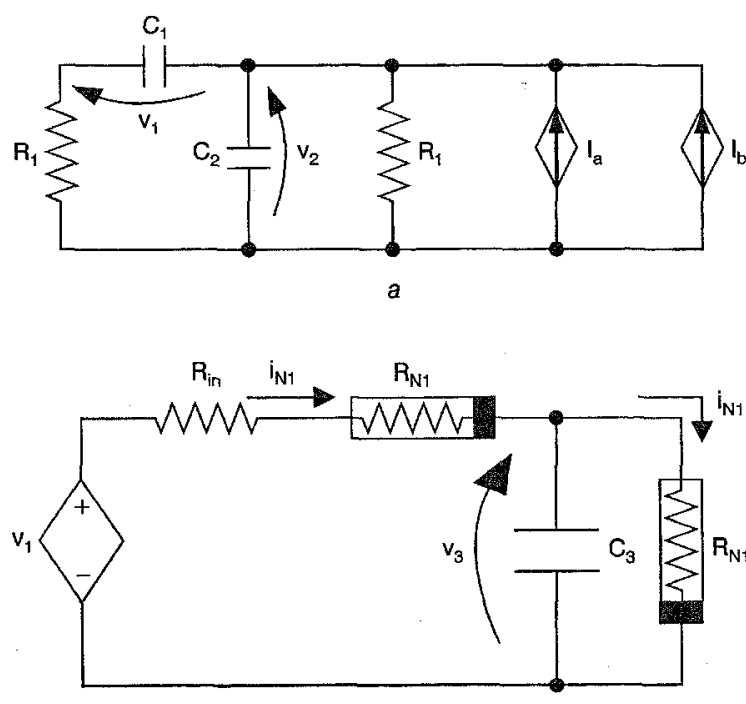

Fig. 1 Chaotic circuit

$a$ Linear part

$b$ Hysteretic part

Circuit description: The considered circuit is shown in Fig. 1. Its upper part is linear, while its lower part is the hysteretic cell. The capacitor voltages $v_{1}, v_{2}, v_{3}$ are the driving variables for the linear voltage controlled current sources (VCCS) $I_{a}$ and $I_{b}$, defined as $I_{a}$ $=G\left(v_{1}+v_{2}\right)$ and $I_{b}=-\left(I_{0} / V_{T 2}\right) v_{3}$, where $G, I_{0}$ and $V_{X 2}$ are proper constants. The linear part of the circuit is identical to its homologue in [1]. Here, the voltage $v_{1}$ drives the cell whose hysteretic behaviour, represented by the capacitor voltage $v_{3}$, influences the linear circuit through $I_{b}$. In [1], this mechanism is obtained via operational transconductance amplifiers (OTA).
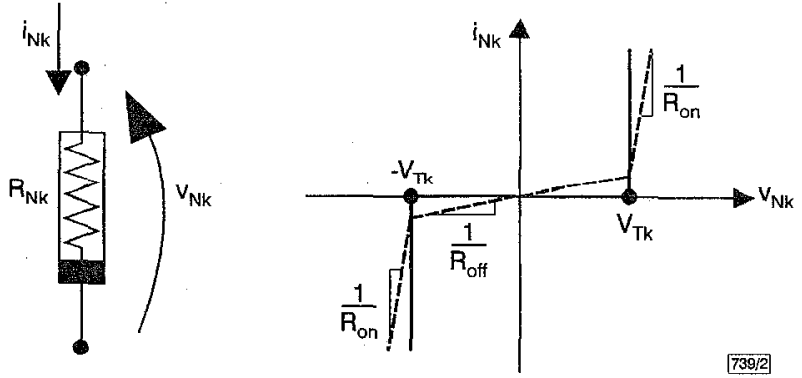

Fig. 2 Two types of $P W L$ characteristics for $R_{N k}(k=1,2)$

\begin{tabular}{l}
--- type $\mathrm{A}$ \\
\hline
\end{tabular}

The hysteresis in the cell is obtained thanks to the nonlinear resistors $R_{N 1}$ and $R_{N 2}$, which have the same type of PWL characteristic. The dashed line, A, shown in Fig. 2, represents a first type 